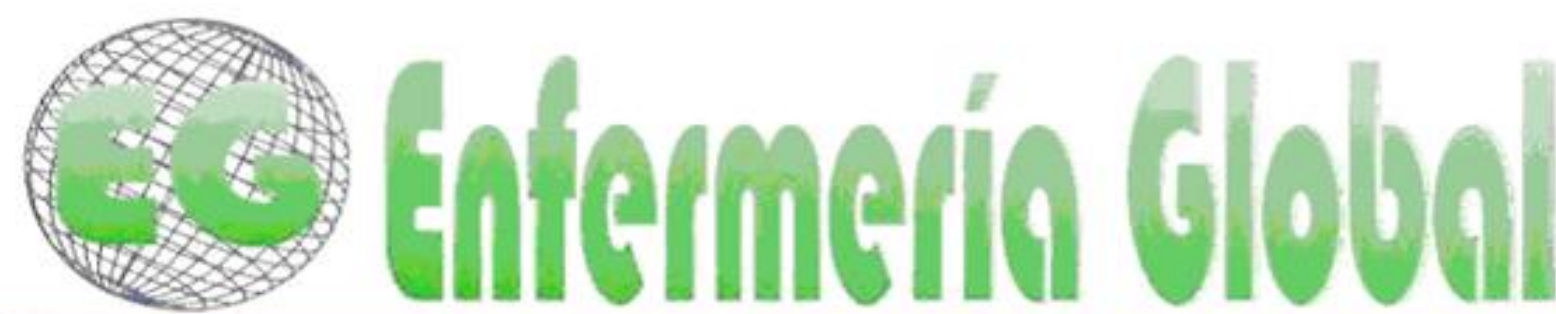

\title{
Valoración del riesgo de caries infantil en un servicio hospitalario de urgencias
}

Risk assessment for childhood caries in a hospital emergency department

*Crovetto Martínez, Rafael ${ }^{* *}$ Ortuzar Otxoa, Leyre *** Martínez Rodríguez, Adelina ${ }^{* * * *}$ Fernández Alonso, Julia ${ }^{* * * * *}$ Escobar-Martínez, Antonio

*Departamento de Estomatología de la Facultad de Medicina y Odontología de la UPV/EHU. Leioa. Vizcaya. **Unidad de Urgencias del Hospital Universitario de Basurto. Bilbao. Vizcaya. ***Departamento de Enfermería de la UPVIEHU. Leioa. Vizcaya. E-mail: adelina.martinez@ehu.es *****Práctica libre. Vizcaya. ****Unidad de Investigación, Hospital Universitario Basurto (Osakidetza) Red de Investigación en Servicios de Salud en Enfermedades Crónicas (REDISSEC), Bilbao, Vizcaya. España.

Palabras clave: Niños; Caries dental; Prevalencia.

Keywords: Children; Dental caries; Prevalence.

\section{RESUMEN}

La caries es una destrucción de los tejidos mineralizados del diente, siendo la enfermedad crónica más prevalente del niño.

Objetivos: Determinar, mediante un estudio de enfermería, la prevalencia de riesgo de caries en los niños que acuden a un Servicio de Urgencias Hospitalarias.

Método: Aplicación del formulario validado para la evaluación de riesgo de caries propuesto por la Sociedad Americana de Odontopediatría, con 14 ítems para niños de más de 7 años y 13 ítems en menores de esa edad. La aplicación de dicho formulario ha contemplado la caducidad de la dentición temporal en relación a la edad del niño estudiado.

Resultados: El formulario se ha completado en 101 niños. El $72.7 \%$ de los niños menores de 7 años y el $89.1 \%$ de los mayores de 7 años presentan uno o más factores de alto riesgo para caries infantil, existiendo diferencias notables en los resultados, en función del origen de los niños o de su etnia. Los peores resultados los ofrecen los niños gitanos cuyo riesgo de caries es el $100 \%$ en grupos de cualquier edad.

Conclusiones: Hemos determinado mediante un estudio de enfermería, la prevalencia de riesgo de caries infantil. La Enfermería española puede y debe asumir un papel activo en la promoción de la salud oral infantil, aplicando activamente sus conocimientos. 


\section{ABSTRACT}

Dental caries is a destruction of the mineralized tissues of the tooth and is the most prevalent chronic disease of children.

Objective: Determine, by a nurse study, the prevalence of high risk of dental caries among children treated at a hospital emergency room.

Methods: We applied an adaptation of the form CAT (caries-risk assessment tool) proposed by the American Society of Pediatric Dentistry, consisting of 14 items for children over 7 years and 13 items for children under that age. The test has been applied considering the resorption time of the deciduous teeth in relation to the child's age studied.

Results: The form has been completed in 101 children. $72.7 \%$ of children under 7 years old and $89.1 \%$ of those aged 7 years have one or more high risk factors for childhood caries. We found differences in the results, depending on the origin or ethnicity of the children studied.

Conclusions: We determined the prevalence of risk for childhood caries. The Spanish nurses can and should play an active role in promoting children's oral health, actively applying their knowledge.

\section{INTRODUCCIÓN}

La caries es una destrucción de los tejidos mineralizados del diente, pudiendo afectar a otras partes de la estructura dental, poniendo en riesgo progresivo a la pieza afectada. La caries dental es la enfermedad crónica más prevalente del niño, superando con creces a enfermedades como el asma infantil y otras ${ }^{1,2}$. Así, la caries afecta prácticamente al $40 \%$ de la población pediátrica occidental aunque alcanza cifras superiores entre los niños de nivel socioeconómico desfavorable ${ }^{3}$. La caries puede causar dolor, dificultad para masticar, alteraciones estéticas y pérdida de piezas dentarias provocando alteración de la masticación, la comunicación verbal e incluso alteraciones psicológicas y académicas ${ }^{4,5}$.

La caries es una enfermedad multifactorial en la que el componente infeccioso es fundamental, lo que la califica de enfermedad transmisible. El Streptococcus mutans es la bacteria cariógena más importante. Otro factor cariógeno es la dieta rica y repetida en carbohidratos fermentables que son hidrolizados por la amilasa salivar hasta la formación de azúcares simples sobre los que el que Streptococcus mutans realiza un metabolismo ácido, que causa el ataque ácido al diente, desmineralizándolo. La mala calidad de su estructura dental o la escasa producción de saliva son también cofactores cariógenos. Otros factores facilitadores de la caries tienen que ver con una pobre higiene oral y una escasa fluorización dental. Un aspecto epidemiológico interesante es que la caries es más frecuente en aquellos niños cuyas madres tienen un deterioro de su dentadura y encías, ya que, entre otras razones, su estado bacteriológico oral es contagioso para sus hijos ${ }^{1-4}$. Los cuidados dentales de los niños, particularmente su higiene bucal, tienen que ver fundamentalmente con la educación sanitaria de los padres, al margen del tipo de cobertura socio-sanitaria a la que estén adscritos.

La introducción en España del Programa de Asistencia Dental Infantil (PADI) ha supuesto un cambio sustancial en la prevención de las caries y sus secuelas. EI PADI se aplicó por primera vez en la Comunidad autónoma del País Vasco y en la Comunidad Foral de Navarra en 1990-1991, pero no fue hasta 15 años más tarde que se extendió a otras comunidades autónomas como Andalucía, Murcia, Aragón y otras $^{6,7}$. La cobertura del PADI es desigual en las comunidades autónomas pero en 
general cubre a los niños entre 7 y 15 años, incluyendo o no a los discapacitados, según regiones ${ }^{7}$. No obstante, la recomendación de la comunidad científica es que el niño debe someterse a evaluaciones de su cavidad oral con el odontólogo antes de los 7 años, para algunos autores al cumplir el primer año de edad ${ }^{8}$, siendo que la asistencia sanitaria del PADI no cubre ese periodo de los primeros 6 años de vida de los niños españoles.

La atención dental se realiza en la consulta odontológica ya que incluye todo el proceso de atención (anamnesis, exploraciones instrumentales y radiológicas, diagnóstico y tratamiento). La Enfermería está excluida del procedimiento, dado el organigrama de este tipo de atención. No obstante, la Enfermería es una de las profesiones sanitarias que más contacto tiene con los niños sanos y enfermos y con sus madres, lo que le permitiría identificar a niños con riesgo epidemiológico elevado para caries dental usando medios propios.

Nuestro objetivo es determinar la prevalencia de alto riesgo de caries en los niños que acuden a un Servicio de Urgencias Hospitalarias.

\section{MATERIAL Y MÉTODO}

Este estudio es prospectivo, transversal y descriptivo y está basado en una encuesta y una exploración. El análisis de datos es descriptivo por medio de frecuencias y porcentajes.

Este estudio se ha realizado en el Servicio de Urgencias de Pediatría del Hospital Universitario de Basurto y cuenta con la aprobación del Comité de Ética de dicho centro.

Criterios de inclusión en el estudio: niños de 2 a 12 años, de ambos sexos, de toda raza, que acuden a urgencias de Pediatría acompañados de su padre o madre, que aceptan participar en el estudio previo consentimiento informado. Son criterios de exclusión la presencia de enfermedad actual grave o muy grave del niño, o cuando la urgencia de la consulta principal implique una dedicación prioritaria a la misma. Igualmente quedan excluidos los niños cuyos padres presenten una situación emocional incompatible con el desarrollo del test o aquellos cuyos padres no firmen el consentimiento informado para realizar el estudio. La propuesta de estudio se ha solicitado a 108 familias con niños atendidos en la Urgencia. La selección de los sujetos ha sido aleatoria.

El formulario aplicado en este estudio para identificar a los niños con alto riesgo epidemiológico de caries dental es el formulario CAT propuesto por la Sociedad Americana de Odontopediatría, que consta de 14 ítems, con sus respectivos niveles de riesgo (Tabla 1$)^{4,8}$. 
Tabla 1-Herramienta CAT para evaluación del riesgo de caries de un niño

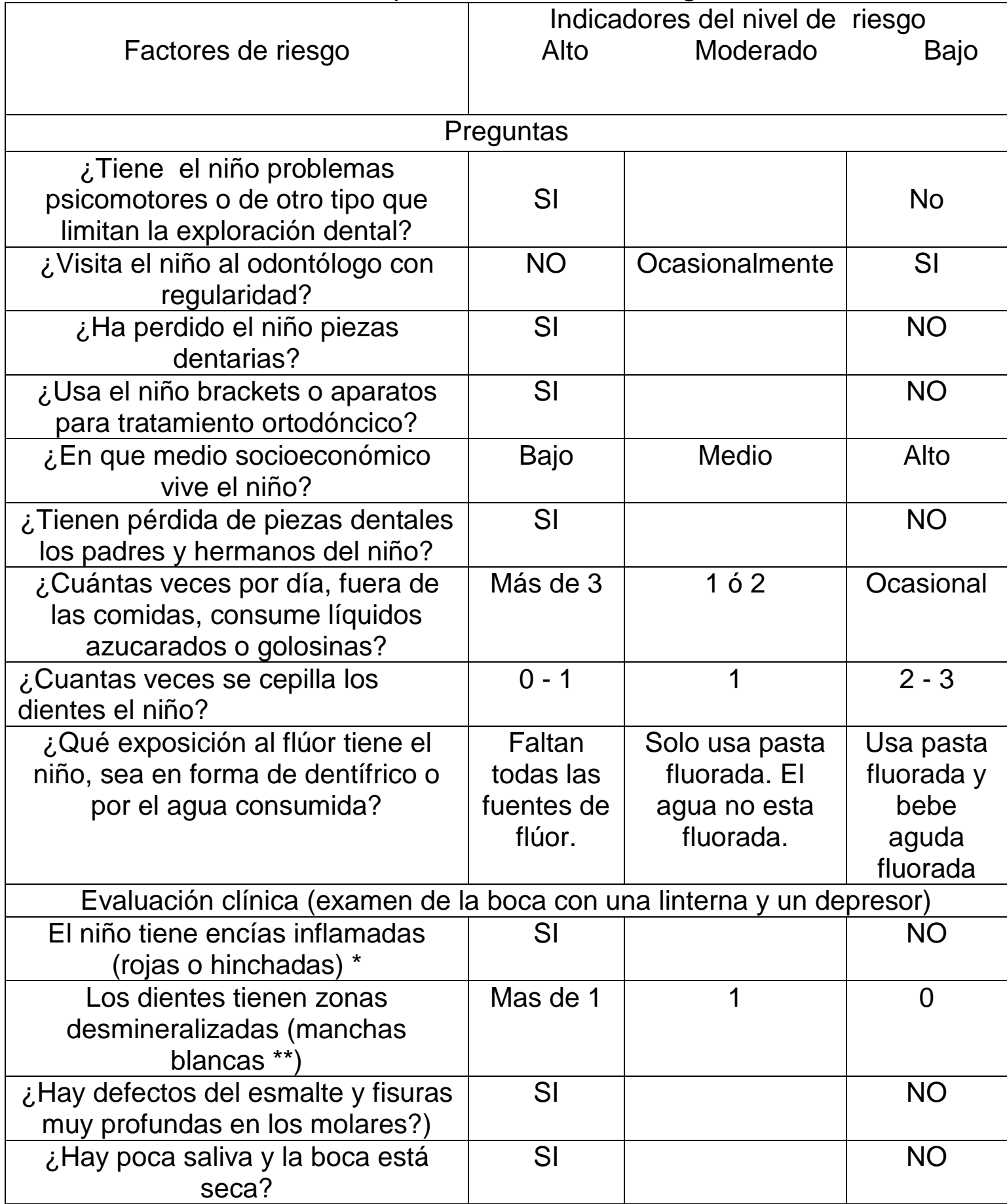

* Una gingivitis se asocia con frecuencia a una mala higiene oral y es un factor de riesgo para caries. ${ }^{* *}$ La caries incipiente puede manifestarse por manchas blancas en el esmalte dental, que traducen la desmineralización incipiente del esmalte. La presencia de surcos opacos, amarillentos o de color café también debe ser considerada como posibles caries incipientes.

El formulario CAT es bastante dinámico y permite adaptaciones a la edad de los niños objeto de estudio. Nosotros hemos aplicado este test con algunas características diferenciales entre los niños mayores y menores de 7 años. En los niños de 7 años 0 más se ha aplicado un formulario CAT de 13 ítems, mientras que en los niños de 2 a 6 años se ha aplicado el mismo formulario pero excluyendo el ítem 2, que hace referencia a la visita periódica al Odontólogo ya que en la Comunidad autónoma del País Vasco el programa PADI se inicia a los 7 años de edad y los padres no tienen constancia de la necesidad de realizar tales visitas antes de esa edad. 
EI CAT consta de dos partes: Anamnesis paternal y exploración oral del niño. Cada una de las preguntas del test y cada una de las exploraciones tienen dos o tres posibles alternativas de respuesta y solo una de ellas es considerada de "alto riesgo cariógeno". La existencia de un solo ítem con alto riesgo cariógeno es bastante para considerar al niño como perteneciente al grupo de "alto riesgo cariógeno".

La mayor parte de las preguntas del formulario CAT son sencillas y no precisan de mayor aclaración. La situación socioeconómica del niño se ha evaluado a partir de los siguientes cuatro datos: Barrio del hábitat (2 niveles posibles: marginales y otros), relación entre superficie habitable y número de miembros de la familia (2 niveles de respuesta: más o menos de 8 metros cuadrados por persona), datos de cohabitación (2 niveles de respuesta (más o menos de 2 niños por habitación), y léxico y cultura aparente de los padres (2 niveles posibles: bajo y otros). Para considerar que el medio socioeconómico en que vive el niño es bajo, y le corresponde un alto factor de riesgo cariógeno, se precisan de 3 de los peores resultados de los 4 evaluados.

La exposición al flúor del niño viene condicionada por su hábitat, siendo que los niños vizcaínos adscritos a este estudio consumen el agua de la red, que está debidamente fluorada. De otro lado, se ha considerado que el uso habitual de pastas dentífricas, casi universalmente fluoradas, es otro factor que asegura una respuesta de bajo riesgo en el niño evaluado, en lo que a fluoración se refiere.

La exploración de la cavidad oral del niño debe contemplar el hecho de que cada pieza de la dentadura temporal del niño tiene fechas aproximadas de aparición y pérdida, por lo que la Enfermera que realiza la exploración considera esta variable al explorar la cavidad oral. Así mismo, la Enfermera, por inspección, con ayuda de depresor y fuente de luz, evalúa la sequedad o correcta hidratación oral, la estructura del esmalte por inspección, así como la situación de la superficie de los dientes (detectar manchas blancas) y la presencia o ausencia de fisuras profundas en los molares. La capacitación de la Enfermera para atender a estas cuestiones ha consistido en su formación odontológica dirigida por el Odontólogo del grupo de investigación.

En el formulario CAT (Tabla I) la existencia de un solo item en la columna "alto" es bastante para considerar al niño como sujeto de alto riesgo cariógeno.

\section{RESULTADOS}

El formulario se ha completado en 101 niños atendidos en la Urgencia. De ellos, 72 son españoles (11 de ellos de etnia gitana), 17 sudamericanos y 12 de otros orígenes geográficos.

La Tabla 2 expone los resultados numéricos y porcentuales de las prevalencias de riesgo de caries en los niños incluidos en el estudio. Se expresan estos valores para tres grupos: todos los niños y por edades. El $72.7 \%$ de los niños menores de 7 años y el $89.1 \%$ de los mayores de 7 años presentan uno o más factores de alto riesgo para caries infantil. Si se consideran todos los niños de la muestra encontramos que el porcentaje de alto riesgo de caries infantil supera el $94 \%$. 
Tabla 2. Resultados numéricos y porcentuales de las prevalencias de riesgo de caries en los niños incluidos en el estudio

\begin{tabular}{|c|c|c|}
\hline $\begin{array}{c}\text { Distribución por edades } \\
\text { (mayor o menor de } \\
\text { 7años) } \\
\text { y total }\end{array}$ & $\mathrm{N}$ (número de niños) & Riesgo elevado \% \\
\hline$<7$ años & 45 & $72.7 \%$ \\
\hline$\geq 7$ años & 46 & $89.1 \%$ \\
\hline Total & 101 & $94.1 \%$ \\
\hline
\end{tabular}

En la Tabla 3 se exponen los resultados numéricos y porcentuales de las prevalencias de riesgo de caries en los niños de diferentes etnias y orígenes geográficos. Se expresan estos valores para tres grupos: todos los niños, y por edades. Se observa variabilidad en los resultados en función del origen de los niños o de su etnia. Así, entre los 61 españoles de etnia no gitana, el riesgo elevado de caries es del $72.7 \%$ en los menores de 7 años edad y del $85.7 \%$ en los de 7 o más años.

Tabla 3. Resultados numéricos y porcentuales de las prevalencias de riesgo de caries en los niños de diferentes etnias y orígenes geográficos.

\begin{tabular}{|c|c|c|c|c|c|c|c|c|}
\hline & \multicolumn{2}{|c|}{ Españoles } & \multicolumn{2}{|c|}{ Etnia gitana } & \multicolumn{2}{c|}{$\begin{array}{c}\text { Sudamerica } \\
\text { nos }\end{array}$} & \multicolumn{2}{c|}{ otros } \\
\hline $\begin{array}{c}\text { Distribución } \\
\text { por edades } \\
\text { y total }\end{array}$ & $\mathrm{n}$ & $\%$ & $\mathrm{n}$ & $\%$ & $\mathrm{n}$ & $\%$ & $\mathrm{n}$ & $\%$ \\
\hline$<7$ años & 33 & $72.7 \%$ & 6 & $100 \%$ & 8 & $62.5 \%$ & 8 & $62.5 \%$ \\
\hline$\geq 7$ años & 28 & $85.7 \%$ & 5 & $100 \%$ & 9 & $100 \%$ & 4 & $75 \%$ \\
\hline Total & 61 & $3.4 \%$ & 11 & $100 \%$ & 17 & $94.1 \%$ & 12 & $91.7 \%$ \\
\hline
\end{tabular}

Los mejores resultados los ofrecen los niños de "otros orígenes" con un $62.5 \%$ y un $75 \%$ de riesgo elevado para caries en niños menores y mayores de 7 años, respectivamente, aunque dado el pequeño número de sujetos que forman este grupo los resultados no tienen significación estadística. Los peores resultados los ofrecen los niños gitanos cuyo riesgo de caries es el 100\% en grupos de cualquier edad.

Un posible beneficio de los niños incluidos en el estudio es el derivado de las recomendaciones que la Enfermera del grupo de estudio hace a los padres del niño, basado en dos pilares: Recomendación de hacer una visita al Odontólogo a la máxima brevedad posible a los padres cuyos hijos hayan sido calificados de alto riesgo cariógeno, y consejos sobre las medidas higiénico-sanitarias adecuadas para cuidar la salud oral, de acuerdo a las recomendaciones científicas internacionales validadas ${ }^{9}$, para lo que se les entregó un folleto explicativo sobre las mismas.

\section{DISCUSIÓN}

La herramienta aplicada en este estudio para la detección de niños con alto riesgo de caries es el test CAT, que fue propuesto para ello por la Sociedad Americana de Odontología Pediátrica en el 2002, siendo actualizado por sucesivas revisiones de dicha Sociedad ${ }^{8-10}$. En el campo de la Enfermería su adopción como instrumento enfermero fue propuesto por Marrs et al ${ }^{4}$. 
Nuestro estudio tiene algunas limitaciones, como es el sesgo que supone la realización del estudio en una urgencia general hospitalaria donde la atención se presta a una población general cuyos recursos de atención sanitaria son los derivados de su cartilla sanitaria del Servicio Vasco de Salud. Esto significa que la muestra en la que se realiza el estudio no puede considerarse representativa de toda la población infantil de la región donde hemos hecho el estudio. De hecho debe considerarse que el $10 \%$ de los niños analizados en nuestro estudio mediante el formulario CAT son de etnia gitana y otro $30 \%$ son foráneos, siendo evidente que esos porcentajes no representan a la población infantil general de Bilbao y Vizcaya. Estamos seguros de que los resultados hubieran sido otros de hacer el estudio en niños que acuden a consultas privadas, odontológicas o pediátricas, periódicamente. Los cuidados dentales de los niños, y su salud dental, tienen que ver fundamentalmente con la educación sanitaria de los padres, al margen del tipo de cobertura socio-sanitaria a la que estén adscritos ${ }^{11,12}$.

Otra limitación del estudio es el hecho de que no contempla la prevalencia de caries real en el colectivo estudiado sino que detecta únicamente la prevalencia del riesgo de presentarlas, sin que podamos demostrar una correlación entre ambas. No obstante existen abundantes trabajos que determinan la incidencia de caries en distintos grupos sociales y en distintos países que nos pueden servir de referencia, especialmente los que se han realizado en nuestra área geográfica. En España, de acuerdo a los estudios epidemiológicos promovidos por el Consejo General de Odontólogos en el 2005, la prevalencia de caries infantil aumenta del 36\% a los 6 años al $45 \%$ a los 12 años 13,14 . Estas cifras resultan similares entre los estudios epidemiológicos comparativos realizados en los últimos años ${ }^{14}$. No obstante, si se considera la prevalencia en los grupos de niños socialmente desfavorecidos de 2 a 5 años las cifras aumentan hasta un $58.7 \%{ }^{15}$. Nuestros peores resultados de riesgo de caries corresponden al grupo de niños gitanos donde encontramos una prevalencia de riesgo del $100 \%$ en todos los grupos de edad. Estas diferencias entre prevalencia de riesgo y prevalencia real tienen su explicación en el valor epidemiológico del test CAT, cuya sensibilidad y valor predictivo negativo son del $100 \%$, en tanto que su especificidad es $2.9 \%$ y su valor predictivo positivo solo alcanza el $49.4 \% 16$. En nuestro estudio hemos encontrado que la prevalencia de riesgo de presentar caries no es menor entre los niños españoles que entre los inmigrantes, lo que contradice la prevalencia real de la caries en estos grupos, de acuerdo con los informes de la Organización Colegial de dentistas de España que señala una prevalencia mayor de caries en los niños inmigrantes que entre los españoles ${ }^{14}$, aunque debe considerarse que nuestra muestra de estudio podría estar sesgada por el alto número de inmigrantes que componen la muestra estudiada. A pesar de estas limitaciones, el formulario CAT es un formulario validado para su aplicación entre personal sanitario no odontólogo, a fin de evaluar el riesgo de caries ${ }^{17}$.

La existencia del programa PADI favorece la mejora de la salud bucal infantil en el conjunto de la población infantil pero solo el $65 \%$ de los niños del País Vasco utilizan el programa PADI y en el resto de España solo alcanza el $40 \%{ }^{14}$, y además, su actuación solo alcanza a niños de entre 7 y 15 años 12,14. No obstante, la recomendación de la comunidad científica es que el niño debe someterse a evaluaciones de su cavidad oral con el odontólogo antes de esa edad ${ }^{8}$.

Entre los Objetivos de salud dental establecidos por el Consejo de Dentistas españoles para el 2020 está el atender a los niños en fase de dentición temporal y potenciar la higiene bucodental apropiada para cada edad estableciendo para ello planes preventivos a conducir en centros escolares. Además, proponen aplicar 
selladores de fosas y fisuras en los niños que presentan alto riesgo de cariogenicidad, lo que implica su identificación previa ${ }^{14}$. De otro lado, aspectos como la supervisión del cepillado dental de los niños por debajo de los 7 años suponen un factor protector para la caries y es muy alto el porcentaje de los niños que no se cepillan o que lo hacen sin supervisión paterna ${ }^{18}$.

Con frecuencia creciente se reclama la implicación activa de los grupos de control sanitario en la prevención de la salud oral infantil en general y de la prevención de la caries en particular ${ }^{4,5,14}$. La enfermería española tiene competencia y oportunidad de ser agente activo en la promoción de la salud oral pediátrica debido a que su formación universitaria superior la faculta para abordar esta tarea de forma competente, igual a como se hace en otros países de nuestro entorno europeo ${ }^{19}$. La actividad enfermera en el centro de salud, debiera incluir dos esferas de actuación: la evaluación de riesgos de caries oral infantil y la educación-promoción sanitaria oral infantil, de acuerdo con las recomendaciones internacionales y nacionales propuestas $^{8,14}$.

\section{CONCLUSIONES}

La caries es la enfermedad más prevalente en el niño. En este trabajo, hemos determinado una elevada prevalencia de riesgo de caries infantil usando los formularios validados para ello, particularmente en el colectivo de niños socialmente desfavorecidos. La Enfermería española debe asumir un papel activo en la promoción de la salud oral infantil, implicándose en la educación y promoción de la misma, ya que es uno de los profesionales sanitarios que más contacto tiene con los niños y sus madres, en las diferentes áreas de su actuación.

\section{REFERENCIAS}

1 Kagihara, LE; Niederhauser, VP; Stark, M. Assessment, managemet, and prevention of early childhood caries. J Am Acad Nurse Pract 2009 Jan;21(1):1-10.

2 Edelstein, BL. The dental caries pandemic and disparities problem. BMC Oral Health 2006 Jun;6 Suppl 1:S2.

3 Hale, Kj. American Academy of Pediatrics section of Pediatrics dentistry. Oral health risk assessment timing and establishment of the dental home. Pediatrics 2003 May;111(5 Pt 1):1113-6.

4 Marrs, JA; Trumbley, S; Malik, G. Early childhood caries: Determining the risk factors and assessing the prevention strategies for nursing intervention. Pediatr Nurs 2011 Jan-Febr;37(1):9-16.

5 Peterson-Sweeney, K; Stevens, J; Optimizing of ealth of infants and children: their oral ealth counts. J Pediatr Nurs 2010 Augus;25(4):244-9.

6 Barriuso Lapresa, Laura; Sanz Barbero, Belen. Análisis multinivel del uso de servicios de salud bucodental por población infanto-juvenil. Gac Sanit. $2011 ; 25(5): 391-396$.

7 Cortés, Francisco Javier. Servicios dentales para jóvenes: el inicio de una andadura. Gac Sanit. 2010 May-Jun;24(3):251-2.

8 American Academy on Pediatric Dentistry. Policy on use of a caries-risk assessment tool (CAT) for infants, children and adolescent (Revised en 2006). Disponible en: http://www.ncdhhs.gov/dph/oralhealth/library/includes/IMBresources/10_P_CariesRiskAssess. pdf 
9 American Academy on Pediatric Dentistry. Policy on early Childhood caries (EEC). Classifications, consequences, and preventive strategies. Pediatr Dent. 20082009;30(Suppl 7):40-3.

10 American Academy of Pediatric Dentistry: Guideline on Caries-risk Assessment and Management for Infants, Children, and Adolescents (Revised 2013). Disponible en: http://www.aapd.org/media/Policies Guidelines/G CariesRiskAssessment.pdf

11 Twtman, S; Fontana, M. Patient risk caries assessment. Monogr Oral Sci 2009;21:91-101.

12 Tapias Ledesma, MA; Jiménez García, R; Carrasco Garrido, P; Lamas F. Gil de Miguel, A: Evolución de la prevalencia de caries en una población infantil de Móstoles entre 1988-1997. Av Odontoestomatol 2005;21(6):333-9.

13 Bravo Pérez, M; Casals, I; Peidró, E; Cortés Martinicorena, FJ; Llodrá Calvo, JC. Encuesta de Salud Oral en España 2005. RCOE 2006; 11(4):409-456.

14 LLodra Calvo, JC. Encuesta de salud oral en España 2010. RCOE 2012;17(1):1341.

15 Mora Leon, L; Martínez Olmos, J. Prevalencia de caries y factores asociados en niños de 2-5 años de los Centros de Salud Almanjáyar y Cartuja de Granada capital. Aten primaria 2000; 26(6):399-404.

16 Yoon, RK; Smaldone, AM; Edelstein, AM. Early childhood caries screening tools: a comparison of four approaches. J Am Dent Assoc 2012 Jul;143(7):756-63.

17 Nainar, SM; Straffon, LH. Predoctoral dental student evaluation of American Academy of Pediatric Dentistry's Caries-Risk Assessment Tool. J Dent Educ. 2006 Mar;70(3):292-5.

18 Vallaizán, C; Aguilar M. Estudio de la prevalencia de la caries y su relación con factores de higiene oral y hábitos tóxicos cariogénicos en escolares. Revista Latinoamericana de Ortondoncia y Odontopediatría. "ortodoncia. Ws edición electrónica Mayo 2012. Disponible en: http://www.ortodoncia.ws/publicaciones/2012/art14.asp

19 Heads, D; Ahn, J; Petrosyan, V; Petersen, H; Ireland, A; Sandy, J. Dental caries in children: a sign of maltreatment or abuse? Nurs Child Young People 2013 Jul;25(6):22-4.

Recibido: 24 de septiembre 2014; Aceptado: 24 de octubre 2014

ISSN 1695-6141

() COPYRIGHT Servicio de Publicaciones - Universidad de Murcia 